ARTICLE

Received 22 Mar 2015 | Accepted 9 Sep 2015 | Published 8 Oct 2015

DOl: 10.1038 /ncomms9589 OPEN

\title{
Planar carbon nanotube-graphene hybrid films for high-performance broadband photodetectors
}

Yuanda Liu ${ }^{1, \star}$, Fengqiu Wang ${ }^{1, \star}$, Xiaomu Wang ${ }^{2}$, Xizhang Wang ${ }^{3}$, Emmanuel Flahaut ${ }^{4,5}$, Xiaolong Liu ${ }^{1}$, Yao Li', Xinran Wang ${ }^{1}$, Yongbing $\mathrm{Xu}^{1}$, Yi Shi ${ }^{1} \&$ Rong Zhang ${ }^{1}$

Graphene has emerged as a promising material for photonic applications fuelled by its superior electronic and optical properties. However, the photoresponsivity is limited by the low absorption cross-section and ultrafast recombination rates of photoexcited carriers. Here we demonstrate a photoconductive gain of $\sim 10^{5}$ electrons per photon in a carbon nanotubegraphene hybrid due to efficient photocarriers generation and transport within the nanostructure. A broadband photodetector (covering $400-1,550 \mathrm{~nm}$ ) based on such hybrid films is fabricated with a high photoresponsivity of $>100 \mathrm{AW}^{-1}$ and a fast response time of $\sim 100 \mu \mathrm{s}$. The combination of ultra-broad bandwidth, high responsivities and fast operating speeds affords new opportunities for facile and scalable fabrication of all-carbon optoelectronic devices.

\footnotetext{
${ }^{1}$ School of Electronic Science and Engineering and Collaborative Innovation Center of Advanced Microstructures, Nanjing University, Nanjing 210093, China. ${ }^{2}$ Department of Electrical Engineering, Yale University, New Haven, Connecticut 06511, USA. ${ }^{3}$ School of Chemistry and Chemical Engineering, 
C ombining low-dimensional nanomaterials into hybrid nanostructures is a promising avenue to obtain enhanced material properties and to achieve nanodevices operating with novel principles ${ }^{1,2}$. The family of carbon allotropes, with its rich chemistry and physics, attracts a great deal of attentions in forming novel hybrid nanostructures ${ }^{2}$. In particular, the excellent electrical conductivities and large specific surface areas of twodimensional (2D) graphene and one dimensional (1D) carbon nanotubes (CNTs) have stimulated earlier theoretical and experimental investigations of $3 \mathrm{D}$ nanotube-graphene hybrid architectures for hydrogen storage ${ }^{3}$, supercapacitors ${ }^{4}$ and fieldemitter devices ${ }^{5}$. Recently, ultrathin CNT layers have been used as a reinforcing component for centimetre-sized chemical vapour deposition (CVD) grown graphene, where hybrid films with enhanced in-plane mechanical strength, uncompromised electrical conductivity and optical transparency are formed ${ }^{6}$. Even more remarkably, the synthesis of covalently-bonded single-wall CNTs (SWNTs) and graphene hybrid film is recently achieved and its use as flexible transparent electrodes is demonstrated ${ }^{7}$. The simple and scalable route for fabricating quasi-2D all-carbon hybrid films is envisioned to offer new opportunities beyond mechanical and nanoelectronic applications ${ }^{2}$.

Both graphene and CNTs exhibit intriguing optical properties, such as broadband and tuneable light absorption, which make them promising materials for photodetectors 8,9 . Graphene has proved excellent for ultrafast and ultrasensitive photodetectors. However, the relatively low absorbance of a single sheet of carbon atoms adversely limits the photoresponsivities of the earlier metal-graphene-metal devices $\left(\sim 10^{-2} \mathrm{~A} \mathrm{~W}^{-1}\right)^{10,11}$. A number of heterogeneous schemes employing plasmonic resonance ${ }^{12,13}$, microcavities $^{14}$, evanescent-wave coupling ${ }^{15}$ and conventional semiconductor nanostructures ${ }^{16}$ have been explored and lifted the photoresponsivity of graphene photodetectors to $\sim 8.6 \mathrm{~A} \mathrm{~W}^{-1}$ (ref. 13). However, these approaches offer only modest responsivity enhancement and introduce fabrication steps that are not manufacturing scalable. Graphene photodetectors based on a photogating mechanism, that is, by depositing a semiconducting quantum-dots (QDs) overlayer, is by far the most superior method in terms of responsivity enhancement and ease of fabrication ${ }^{17,18}$. But the spectral coverage is limited by the absorption range of the QDs and enhancement is effective only at extremely low light intensities (that is, pico-Watt or $10^{-12} \mathrm{~W}$ ) due to the use of exfoliated graphene flakes (lateral size $\sim 5 \mu \mathrm{m})^{16}$. Although an astonishing gain-bandwidth product of $\sim 10^{9}$ has been demonstrated ${ }^{16}$, such systems are intrinsically slow with electrical bandwidth around $1-10 \mathrm{~Hz}$, as limited by the low carrier mobility and long carrier recombination time of the QDs ${ }^{17}$.

SWNTs, on the other hand, are $\pi$-conjugated, 1D structures with nanometre diameters ${ }^{19}$. They exhibit either metallic or semiconductor features depending on the tube chiralities ${ }^{20}$. Both metallic and semiconducting SWNTs are effective light absorbers in a wide spectral range ${ }^{21}$ and intrinsic mobility in semiconducting SWNTs is estimated to be as high as $10^{5} \mathrm{~cm}^{2} \mathrm{~V}^{-1} \mathrm{~s}^{-1}$ at room temperature ${ }^{22}$. Due to $1 \mathrm{D}$ quantum confinement, photoexcitations in SWNTs demonstrate rich physics ${ }^{9}$. For example, localized excitons with large binding energy is the main excitation for low-energy transitions (that is, $S_{11}$ ) in semiconducting SWNTs ${ }^{23}$, while metallic tubes can act as an efficient charge transport channel for SWNT ensembles to adjacent conducting media ${ }^{24}$. Although extensive efforts have been directed towards the realization of nanotube photodetectors, the weak photoresponse $\left(<10^{-3} \mathrm{AW}^{-1}\right)$ of single-tube photovoltaic devices greatly limits their practical use ${ }^{25}$, while nanotube bolometric detectors are known to suffer from slow response time ${ }^{26}$. Recently, efficient charge transfer has been identified at junctions formed by graphene and SWNTs ${ }^{24}$. The intimate electronic coupling between the two $\mathrm{sp}^{2}$-hybridized carbon allotropes, combined with the various strategies available for structural and chemical engineering of the interfacial electronic properties ${ }^{27,28}$, make such all-carbon hybrid an excellent candidate in enabling phototransistors with balanced and tuneable gain-bandwidth characteristics.

Here we demonstrate a proof-of-concept photodetector based on a planar atomically thin SWNT-graphene hybrid film. In our design, enhanced broadband light absorption is achieved in the hybrid film. Compared with pristine graphene photodetectors which exhibit only weak Schottky junction and high Auger recombination rate, the large built-in potential at the $1 \mathrm{D}-2 \mathrm{D}$ interface promotes effective separation of electron-hole pairs and reduces recombination of spatially isolated photocarriers. Furthermore, the trap-free interface enables a relatively fast operation rate. The devices exhibit a significant photoconductive gain of $\sim 10^{5}$, together with a high electrical bandwidth of $\sim 10^{4} \mathrm{~Hz}$ (response time $\sim 100 \mu \mathrm{s}$ ) across visible to nearinfrared range $(400-1,550 \mathrm{~nm})$. The reported device constitutes a first implementation of large-area, quasi-2D SWNT-graphene hybrid film for optoelectronic devices and is envisaged to be important for optical communication, spectroscopy, remote sensing and high-resolution imaging applications. Equally important, we demonstrate for the first time the possibility of harvesting robust excitons widely supported in 1D systems by a 2D layered material. Our results not only open up new avenues for studying fundamental carrier transport and relaxation pathways in nanometre-scale, $1 \mathrm{D}$ van der Waals junctions, but also pave the way for constructing high-performance optoelectronic heterostructures by planar 1D-2D hybrid building blocks, other than the use of purely $2 \mathrm{D}$ layered materials.

\section{Results}

SWNT-graphene phototransistor fabrication. We fabricated proof-of-concept phototransistors using the SWNT-graphene hybrid film, as illustrated schematically in Fig. 1a. The degenerately n-doped Si substrate with $285 \mathrm{~nm}$ thermal oxide was used as the back-gate. Wafer-scale SWNT-graphene hybrid film is fabricated by transferring CVD grown graphene onto an ultrathin layer of SWNTs formed on the $\mathrm{SiO}_{2} / \mathrm{Si}$ substrate (see Methods). Figure $1 b, c$ shows a representative tapping mode atomic force microscope (AFM) image of the hybrid film, where nanotubes form filament structures similar to the vein-like support as in ref. 6. Figure 1d shows the height profile along the red line in Fig. 1b, where unbundled SWNTs with diameters in the range of $1.0-1.6 \mathrm{~nm}$ are observed. This is in agreement with a mean tube diameter of $\sim 1.4 \mathrm{~nm}$ as inferred from the Radial Breathing Mode position of the Raman measurement (Supplementary Note 1, Supplementary Fig. 1). To investigate the physical characteristics of the junctions formed at graphene and SWNTs, a section of graphene is mechanically removed using a tape stripe (dark brown area, Fig. 1c), so that the height of the SWNT-graphene junction with respect to the substrate can be directly measured. This yields a height of $\sim 2.2 \mathrm{~nm}$ for the SWNT-graphene junction (Fig. 1e), while the isolated SWNT height as measured from the uncovered portion indicates a height of $1.2 \mathrm{~nm}$ (Supplementary Fig. 2). The height of graphene on $\mathrm{SiO}_{2} / \mathrm{Si}$ substrate is measured to be $\sim 0.8 \mathrm{~nm}$, indicative of single atom layer ${ }^{29}$. The inferred offset at the SWNT-graphene interface is $\sim 0.6 \mathrm{~nm}$, which is larger than the interlayer distance of graphite $(\sim 0.335 \mathrm{~nm})$, providing evidence for a long-range van der Waals interactions ${ }^{30}$. Figure if shows the optical absorption spectrum of the graphene and the SWNT-graphene hybrid film, illustrating enhanced broadband absorption due to the incorporation of 
d
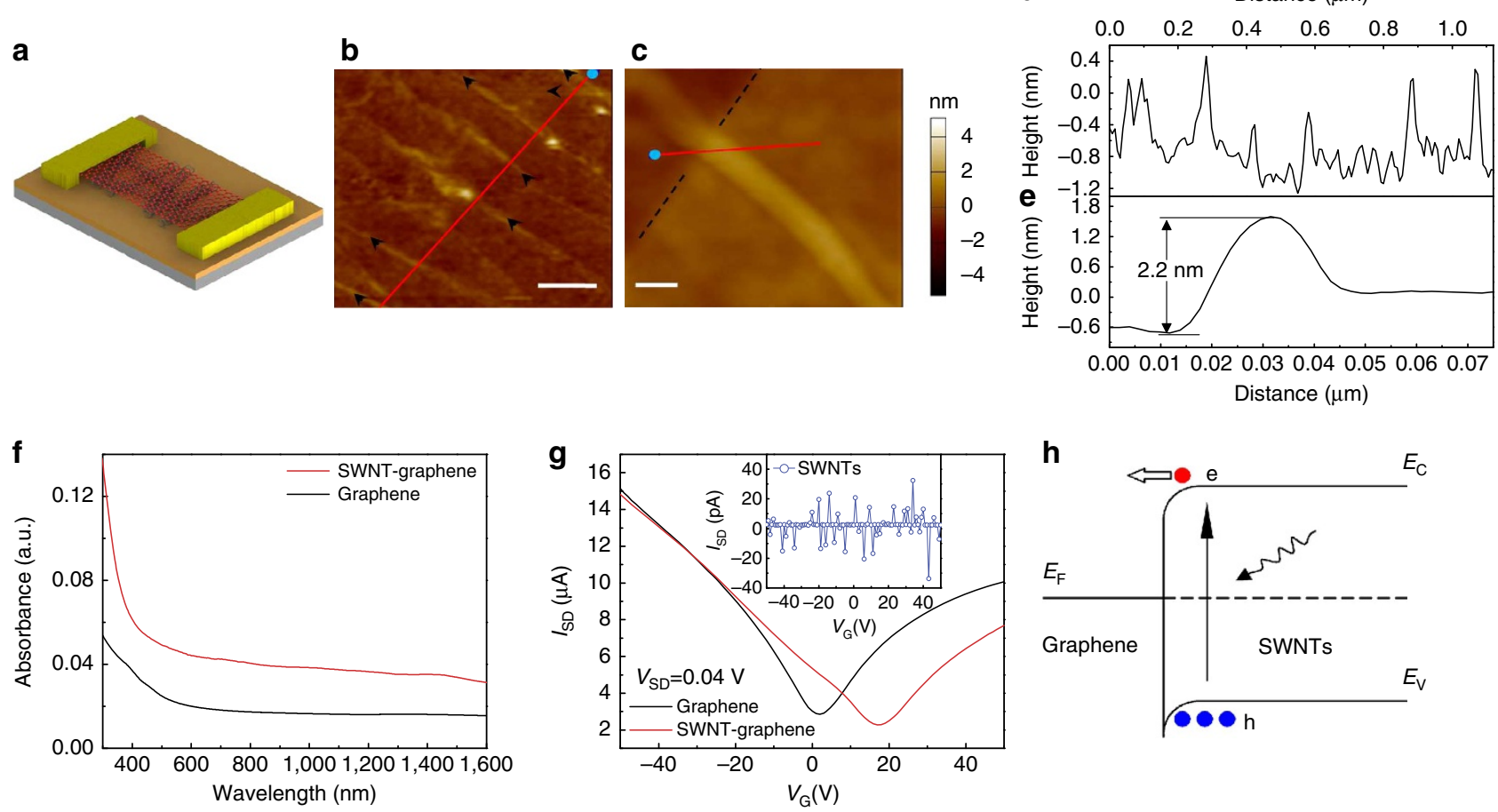

Figure 1 | Planar SWNT-graphene hybrid film and photodetector. (a) Schematic of the phototransistor. (b) $\mathrm{AFM}$ image of the hybrid film on the $\mathrm{SiO} / \mathrm{Si}$ substrate (Scale bar, $200 \mathrm{~nm}$ ). Black arrows are used to pinpoint individual SWNT. (c) AFM image of one individual SWNT partially covered by graphene (Scale bar, $20 \mathrm{~nm}$ ). SWNT was uncovered by mechanically exfoliating the top graphene using a tape stripe. The black dashes show the edge of the graphene (left: $\mathrm{SiO}_{2}$; right: graphene). (d) Height profile along the red line in $\mathbf{b}$. The blue dot in $\mathbf{b}$ marks the zero point in $\mathbf{d}$. (e) Comparison of the respective height profiles of the graphene-covered portion and the uncovered portion of SWNT shown in panel $\mathbf{c}$. (f) Ultraviolet-visible-infrared absorbance curves of graphene and SWNT-graphene hybrid film on quartz. (g) Transfer characteristics of the graphene, SWNTs and SWNT-graphene transistors without light. Comparing with the graphene transistor, the Dirac point of the SWNT-graphene transistor shifted from 2 to $17 \mathrm{~V}$, indicating p-type doping in the graphene sheet induced by SWNTs. The electron (hole) mobility decreases from $6,142 \mathrm{~cm}^{2} \mathrm{~V}^{-1} \mathrm{~s}^{-1}\left(7,146 \mathrm{~cm}^{2} \mathrm{~V}^{-1} \mathrm{~s}^{-1}\right)$ to $3,771 \mathrm{~cm}^{2} \mathrm{~V}-1 \mathrm{~s}-1$

$\left(4,666 \mathrm{~cm}^{2} \mathrm{~V}^{-1} \mathrm{~s}^{-1}\right)$. The inset exhibits that the source-drain current of the SWNTs transistor is $<10^{-11} \mathrm{~A}$, indicating disconnected electric pathways for the pristine SWNTs channel. (h) The energy band diagram at the junction formed by graphene and semiconducting SWNTs. Photogenerated electrons in SWNTs are transferred to graphene due to the built-in field at the junction.

SWNTs. The $\mathrm{S}_{11}$ and $\mathrm{S}_{22}$ bands are found to be located at $\sim 1,800$ and $\sim 1,000 \mathrm{~nm}$ with higher SWNTs loadings (Supplementary Fig. 3), which agree well with the tube diameter distributions.

To probe the electrostatic doping scenario at the $1 \mathrm{D}$ van der Waals junctions formed by graphene and SWNTs, transfer curves of the devices before and after forming the hybrid film are compared. It is observed that the Dirac point of the hybrid transistor shifted from 2 to $17 \mathrm{~V}$, indicating p-type doping of the graphene sheet by the SWNTs layer. It should be pointed out that due to the electronic inhomogeneity of the SWNTs, the electrostatic doping of graphene is expected be an overall effect from both metallic and semiconducting SWNTs. As metallic SWNT is found to form Ohmic contact with graphene ${ }^{31}$ (that is, there is no $\mathrm{p}-\mathrm{n}$ junction or Schottky barrier), photoresponse of the hybrid channel is expected to arise from semiconducting SWNTs with suitable band alignment with graphene. Figure $1 \mathrm{~h}$ provides a representative band alignment that supports the electrostatic-doping scenario at the graphene semiconducting SWNT interface (where constituent semiconducting SWNTs have work functions larger than that of graphene), that is, photogenerated electrons transport from SWNTs to graphene under the built-in field at the SWNT-graphene junction. In addition, the relatively low contact resistance for SWNTgraphene junctions $(<0.1 \mathrm{M} \Omega)^{31}$ as compared with those at SWNT-metal $(1-4 \mathrm{M} \Omega)^{32,33}$ and SWNT-SWNT contacts $(>2 \mathrm{M} \Omega)^{34,35}$ may further encourage photocarriers transport between SWNTs and graphene.
Photoresponse under visible light illumination. The real-optical microscope image of the SWNT-graphene photodetector is shown in the inset of Fig. 2a. No cracks or wrinkles are discernible. It is worth noting that in fabricating our device the SWNT layer is placed beneath graphene to ensure the formation of high quality SWNT-graphene interface, that is, to avoid degradation caused by the residual poly(methyl methacrylate) used in the graphene transfer procedures ${ }^{36}$. Photoresponse of the SWNT-graphene hybrid device to $650 \mathrm{~nm}$ visible light is measured. Figure 2a shows a set of source-drain currents $\left(I_{\mathrm{SD}}\right)$ as a function of the back-gate voltage $\left(V_{\mathrm{G}}\right)$ under different illumination levels, where continuous negative shift of the voltage for Dirac point (the charge neutrality point) is observed, confirming a photocurrent generation mechanism as depicted by Fig. 1h. Control devices with only SWNT layer as the channel are found to be insulating and exhibit no detectable photoresponse, probably due to the low density of nanotubes on the substrate (Inset of Fig. 1g). The on/off ratio is about 4, suggesting graphene is the primary conductive channel ${ }^{37}$. For $650 \mathrm{~nm}$ light $(1.9 \mathrm{eV})$ with photon energies larger than the $\mathrm{S}_{22}$ bandgap of the semiconducting SWNTs $(\sim 1.2 \mathrm{eV})$, free electrons can be directly excited from the valence band to the conduction band. Photogenerated electrons in SWNTs are transferred to the graphene channel due to the built-in electric field, while the holes are trapped in the SWNTs due to the potential barrier at the interface. The conductance of the graphene channel decreases for $V_{\mathrm{BG}}<V_{\mathrm{D}}$ where the carrier transport is hole-dominated, but 
a

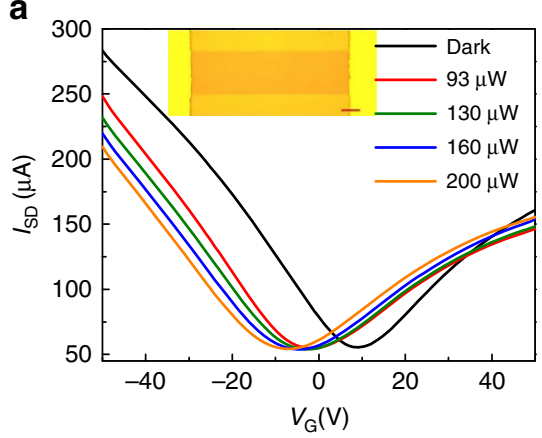

C

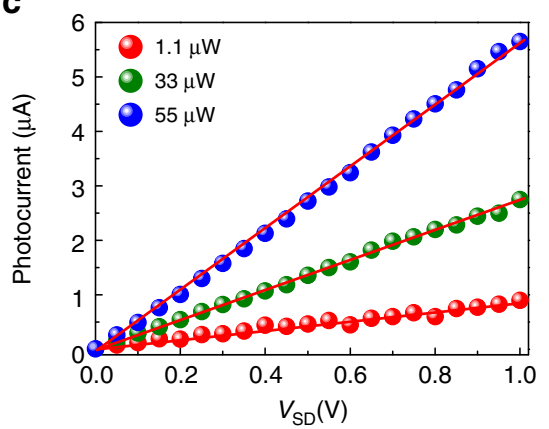

b

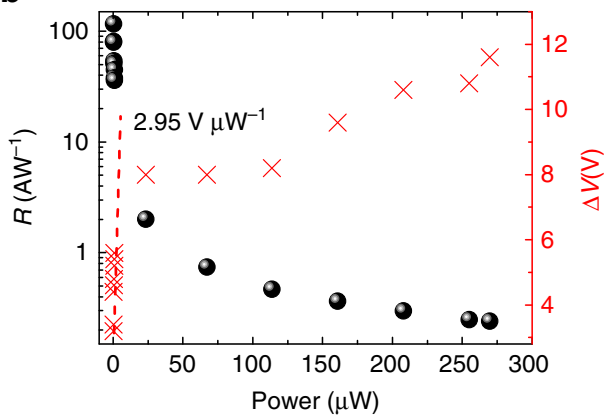

d

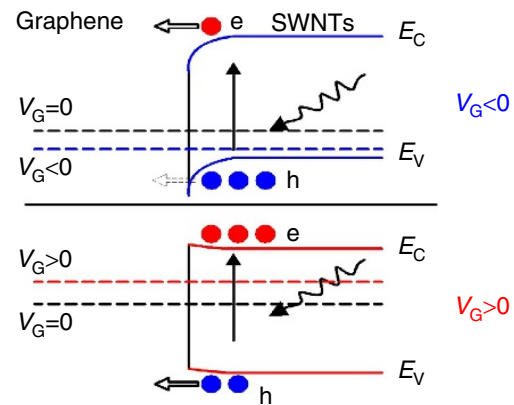

Figure 2 | Photoresponse performance under $\mathbf{6 5 0} \mathbf{~ n m ~ i l l u m i n a t i o n . ~ ( a ) ~ S o u r c e - d r a i n ~ c u r r e n t ~}\left(I_{S D}\right)$ as a function of back-gate voltage $\left(V_{G}\right)$ for the $S W N T-$ graphene device with increasing $650 \mathrm{~nm}$ illumination intensities. $V_{\mathrm{SD}}=0.5 \mathrm{~V}$. Increasing of the illumination leads to a photogating effect that shift the Dirac point to lower $V_{\mathrm{G}}$, indicating electron doping of the graphene sheet. The inset is the optical micrograph of the fabricated device (Scale bar, $10 \mu \mathrm{m}$ ). The gold areas indicate the metal electrodes. The orange area and $\mathrm{LT}$ orange areas are graphene channel and $\mathrm{SiO}_{2} / \mathrm{Si}$ substrate, respectively. (b) Responsivity (black dots) and shift of Dirac point (red cross) as a function of the $650 \mathrm{~nm}$ illumination. (c) The magnitude of the photocurrent increases linearly with sourcedrain bias voltage $\left(V_{\mathrm{SD}}\right)$ for different optical powers $\left(V_{\mathrm{BG}}=0\right)$. Red lines are linear fits. (d) Energy-band diagram of the SWNT-graphene phototransistors at different back-gate voltage. The dash lines correspond to the Fermi level of graphene at different gate voltages. The blue and red lines schematically illustrate the gate voltage dependence of the SWNT energy levels.

increases for $V_{\mathrm{BG}}>V_{\mathrm{D}}$, where carrier transport is electron dominated. Figure $2 \mathrm{~b}$ plots the negative shift of the Dirac point voltage $(\Delta V)$ as a function of the illumination power, clearly revealing the high sensitivity of the SWNT-graphene device. $I_{\mathrm{SD}}$ as a function of $V_{\mathrm{G}}$ under different illumination power of $650 \mathrm{~nm}$ demonstrates effective tuning of the photocurrent and the photoresponsivity with the back-gate voltage, where a maximum photocurrent of $>90 \mu \mathrm{A}$ is obtained at a negative $V_{\mathrm{BG}}$ around $-20 \mathrm{~V}$ (Supplementary Fig. 4a). In Fig. 2c, linear scaling of the photocurrent with source-drain bias voltage $\left(V_{\mathrm{SD}}\right)$ for different optical powers at $V_{\mathrm{BG}}=0$ is clearly observed. Figure $2 \mathrm{~b}$ also plots the photoresponsivity as a function of the illumination power. To faithfully illustrate the performance of the device we define the responsivity using the incident power rather than the absorbed power. At an illumination power of $\sim 0.2 \mu \mathrm{W}$, the photodetector exhibits a drastically enhanced responsivity of $\sim 120 \mathrm{~A} \mathrm{~W}^{-1}$, as compared with a bare graphene device $\sim 10^{-2} \mathrm{~A} \mathrm{~W}^{-1}$. Since the responsivity does not show sign of saturation, higher photoresponse on the order of $\sim 1,000 \mathrm{~A} \mathrm{~W}^{-1}$ is expected at lower excitation power levels (Supplementary Fig. $4 \mathrm{~b})$.

To rule out potential contributions from thermal effects, including bolometric contribution from the SWNT layer ${ }^{26}$ and photothermoelelectric contribution from the metal electrodes ${ }^{38}$, we measured the $I_{\mathrm{SD}}-V_{\mathrm{G}}$ at temperatures from 53 to $273 \mathrm{~K}$ (Supplementary Fig. 5 and Supplementary Note 2). Source-drain current increases with an increasing temperature from 53 to $173 \mathrm{~K}$, and a slight decrease in the range $173-273 \mathrm{~K}$, as expected for SWNT response ${ }^{26}$. The rather small temperature dependence of $I_{\mathrm{SD}}$ allows us to rule out resistance increase caused by heating of SWNTs as the mechanism for photocurrent generation. Light induced heating of source or drain metal contacts is reported to lead to a temperature gradient, resulting in a photothermoelectric contribution to the photocurrent ${ }^{38}$. However, we did not observe detectable photocurrent in our devices by illuminating visible light $(650 \mathrm{~nm})$ on either metal contact. Therefore, the photoresponse of our device is ascribed to a photogating effect, where channel resistance is modulated through capacitive coupling caused by trapped photogenerated charges.

We further verify the operation mechanism of our devices by correlating the gate dependent photocurrents with the band alignment at the SWNT-graphene junctions. As schematically illustrated in Fig. 2d, incident photons excite ground-state electrons of SWNTs into excited states, and then electron-hole pairs are formed at the SWNT-graphene interface. For $V_{\mathrm{G}}<0$, the Fermi level of graphene is lowered, which leads to a steeper upward band bending and an enhanced built-in electric field at the SWNT-graphene junction. This facilitates more photoelectrons to transfer from SWNTs to graphene, leading to an increasing photocurrent. As the magnitude of negative $V_{\mathrm{G}}$ continues to increase, the trapped holes in the valence band of SWNTs begin to tunnel through the thinned barrier into the graphene channel, resulting in a decreased photocurrent. In the case of $V_{G}>0$, the Fermi level of graphene is raised up, the potential offset for electrons is effectively decreased. Therefore, the photocurrent at a given positive gate voltage is smaller than that for a negative gate voltage of equal amplitude. When $V_{\mathrm{G}}$ is sufficiently high, the SWNT energy band will flip to bend downwards, allowing photogenerated holes to transfer to the 
a

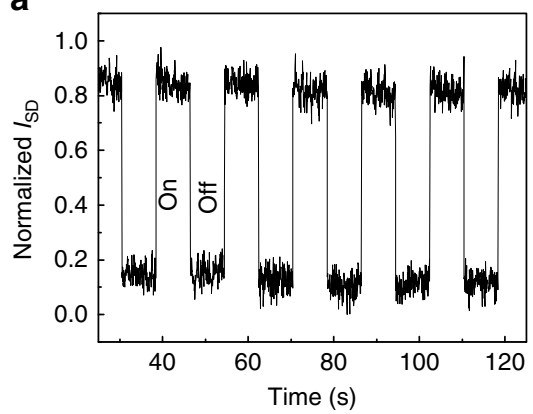

b

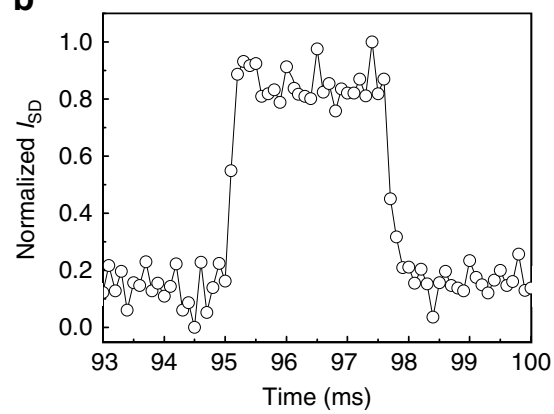

c

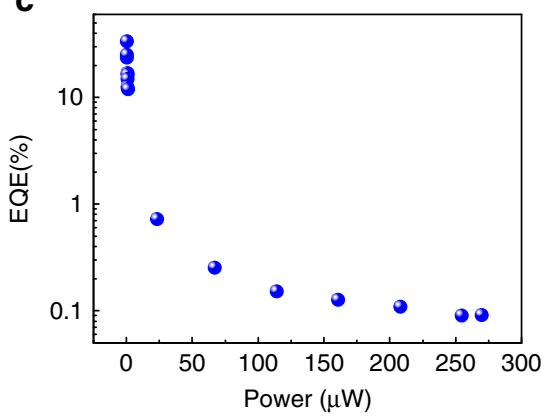

d

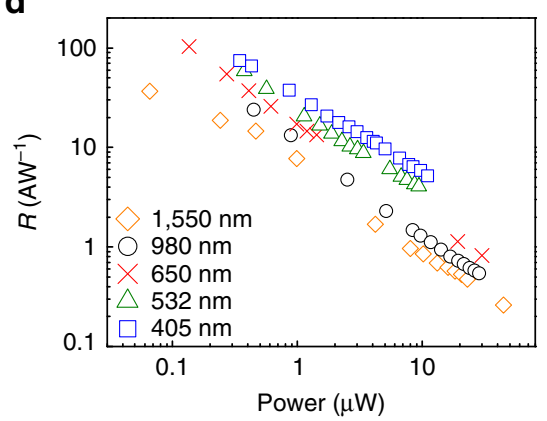

e

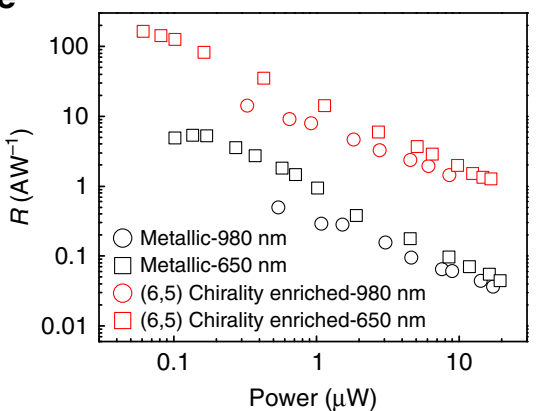

Figure 3 | Temporal characteristics and ultra-broadband photoresponse. (a,b) Temporal photocurrent response of the SWNT-graphene hybrid photodetector, indicating a rise time and a fall time on the order of $\sim 100 \mu$ s. The illumination power is $440 \mu \mathrm{W}$ and the laser wavelength is 650 nm. (c) External quantum efficiency as a function of illumination power at $650 \mathrm{~nm}$. (d) Responsivities as a function of the optical power for different illumination wavelengths $(405,532,650,980$ and 1,550 nm). (e) Comparison of responsivities measured from devices using purified metallic- and (6,5) chiralityenriched SWNTs. It is shown that the responsivity of the metallic tube-based devices is $\sim 5 \%$ of that of $(6,5)$ chirality tube-based devices.

graphene channel. This is the reason why the conductivity decreases for $V_{\mathrm{G}}>40 \mathrm{~V}$, and the photocurrent subsequently switches sign (Supplementary Fig. 4a).

Fast response time. Response time is another key figure of merit for photodetectors and is also relevant in revealing the physical mechanism of the device operation. Figure $3 \mathrm{a}$ shows the on/off source-drain current of the photodetector at an incident power of $440 \mu \mathrm{W}\left(650 \mathrm{~nm}, V_{\mathrm{SD}}=1.2 \mathrm{~V}\right)$. The temporal photoresponse was measured at $173 \mathrm{~K}$ to suppress the outside interference including scattering centres from the substrate and charge-trapping surface states $^{31}$. After hundreds of on-off cycles, photocurrent level is well retained, demonstrating good reliability and reversibility of our devices. At the same experimental conditions, we did not observe any detectable photocurrent in a control device with a bare graphene channel. Figure $3 \mathrm{~b}$ gives the high-resolution temporal response (sampling interval $100 \mu \mathrm{s}$ ). To encourage photocarriers separation we apply a $5 \mathrm{~V}$ source-drain voltage. Sharp responses are observed from which we estimate the rise time and the fall time to be $\sim 100 \mu$ s. The operation speed of our device is $>1,000$ times faster than a graphene-QDs detector with similar photosensitive area ${ }^{17}$, and can be further reduced by scaling the device dimensions. The high mobility and fast transfer of carriers within the SWNT-graphene hybrid film are believed to account for the excellent temporal performance of our devices.

The EQE (External Quantum Efficiency) was estimated using the parallel plate capacitor $\operatorname{model}^{18}, \mathrm{QE}=\Delta V \times C_{\mathrm{ox}} / \phi_{\text {photon, }}$ where $\Delta V$ is the Dirac voltage shift, $C_{\mathrm{ox}}$ is the capacitive coupling of the back-gate for $285 \mathrm{~nm}$ thick thermal oxide $\left(7 \times 10^{10} \mathrm{~cm}^{-2} \mathrm{~V}^{-1}\right), \phi_{\text {photon }}$ is the photon flux. As shown in Fig. 3c, for $650 \mathrm{~nm}$ light our devices show a power-dependent EQE ranging from 34 to $12 \%$ for power illumination $<1 \mu \mathrm{W}$, and an EQE of $<0.7 \%$ with incident power $>25 \mu \mathrm{W}$. The peak value for the QE is slightly higher than the $25 \%$ achieved with QDs ${ }^{18}$, demonstrating the high efficiency of photocarriers generation and transport in the SWNTs-graphene hybrid. From the transfer curve measured in the absence of light, it is estimated by the Kim model $^{39}$ that the field-effect mobility for electrons and holes are 3,920 and $3,663 \mathrm{~cm}^{2} \mathrm{~V}^{-1} \mathrm{~s}^{-1}$, respectively. This is superior to graphene sensitized with $\mathrm{PbS} \mathrm{QDs}^{18}$. Such high mobilities confirm that graphene can be doped by $1 \mathrm{D}$ SWNTs, yet preserve its outstanding electronic properties, facilitating high gain and fast response time. The carrier transit time of our device is estimated to be on the order of $10^{-9} \mathrm{~s}$ (based on a carrier mobility of $\sim 3,920 \mathrm{~cm}^{2} \mathrm{~V}^{-1} \mathrm{~s}^{-1}$, a channel length of $70 \mu \mathrm{m}$ and a bias of $5 \mathrm{~V}$ ), thus the photoconductive gain $G=\tau_{\text {lifetime }} / \tau_{\text {transit }}$ (the ratio of the lifetime of the trapped holes over the electron transit time from source to drain) is on the order of $\sim 10^{5}$ using a trapped carrier lifetime of $\sim 100 \mu \mathrm{s}$. Therefore the gainbandwidth product of our SWNT-graphene device is on the order of $1 \times 10^{9} \mathrm{~Hz}$ (given by the product of $\sim 10^{5}$ gain and $\sim 10^{4} \mathrm{~Hz}$ of bandwidth), which is quantitatively similar to the $\mathrm{PbS}$ QD-graphene hybrid photodetector ${ }^{18}$.

Ultra-broadband photoresponse. Characteristics of broadband photoresponse of our device over a range of incident wavelengths is investigated by using multiple laser diodes operating from visible to the near-infrared range $(405,532,650,980$ and $1,550 \mathrm{~nm})$. The responsivities for various wavelengths are summarized in Fig. 3d, where ultra-broadband spectral coverage is observed. Higher responsivities were obtained at lower incident power levels. At a moderate illumination power of $\sim 0.3 \mu \mathrm{W}$, all wavelengths exhibit a responsivity $>10 \mathrm{~A} \mathrm{~W}^{-1}$, a typical specification for commercial photodetectors. The responsivity value is 
higher for shorter wavelengths than for longer ones (Supplementary Fig. 6), consistent with the absorption spectrum of the SWNTs used, which is the usual case for phototransistors based on a photogating mechanism ${ }^{18}$. From the wavelength dependence, even higher photoresponsivities are expected for ultraviolet wavelengths.

\section{Discussion}

The essential ingredients of our devices are the sensitivity to external electrostatic in graphene conductivity, the broadband and tuneable photon absorption in both graphene and SWNTs, and the high-speed transportation of photocarriers in the planar SWNT-graphene hybrid. To resolve the impact tube diameters and chiralities may have on the photodetection processes, we fabricated control devices using highly purified metallic tubes and $(6,5)$ chirality-enriched semiconducting SWNTs. Quite different photodetection mechanisms were revealed (Supplementary Figs 7 and 8, Supplementary Note 3). Figure $3 \mathrm{e}$ compares the photoresponsivities of purified metallic and semiconducting SWNTs-based devices, and the responsivity of the metallic tube-based devices is seen to be $\sim 5 \%$ of that of $(6,5)$ chirality tube-based devices. The results clearly identify semiconducting tubes as the main contribution for photocurrents. In the meantime, unlike nanoelectronic devices where distinct SWNT chirality is desirable, our devices will not degrade by the presence of multiple species of SWNTs, similar to SWNT-based nonlinear absorption devices ${ }^{40}$. The mitigation of requirements for tube chiralities also facilitates the practical and scalable fabrication of such films. The performance of the proof-of-concept photodetector based on SWNT-graphene hybrid film can be further optimized by multiple strategies including controlling the density, chiralities ${ }^{41}$ and alignment direction of the nanotube layer and by using refined SWNT-graphene hybrid film fabrication methods 6,7 .

In summary, by combining large-area CVD grown graphene with atomically thin SWNT layer, we have formed a quasi-2D allcarbon hybrid film, which exhibits strikingly enhanced photodetection capabilities superior to either graphene or SWNTs. In contrast to previous charge trap based phototransistors, such hybrid film based photodetectors exhibit not only a significant photoconductive gain of $\sim 10^{5}$, but also a fast response time of $\sim 100 \mu \mathrm{s}$ and an ultra-broadband sensitivity across visible to near-infrared, covering the telecommunication band at $\sim 1.5 \mu \mathrm{m}$. Benefiting from the solution processability of SWNTs, large-scale growth and transfer of graphene, as well as compatibility with standard photolithography, our devices exhibit great potential for use in photodetection applications requiring large sensing area, high photoresponsivities, video-frame-rate processing and substrate flexibility. The results demonstrated here represent a significant step towards facile and scalable fabrication of highperformance optoelectronic devices using all-carbon hybrid nanostructures and have substantial implications for fundamental investigation of the van der Waals interactions between layered materials and in-plane quantum wires, in the $1 \mathrm{D}$ limit.

\footnotetext{
Methods

Phototransistor fabrication. The graphene samples were grown on copper foil by CVD method, and Raman spectroscopy combined with optical microscope characterizations point to a defect-free single-layer sample. We use single-wall CNTs (SWNTs) from a commercial supplier (Carbon solutions Inc.). The phototransistors are fabricated as follows: SWNTs suspensions are produced by tipsonicating $1 \mathrm{mg}$ nanotube soot in $10 \mathrm{ml}$ NMP ( $N$-methyl-2-pyrrolidone). The resultant suspensions are centrifuged with $10,000 \mathrm{~g}$ for $1 \mathrm{~h}$ before the supernatant is collected for the fabrication of SWNTs thin film on $\mathrm{SiO}_{2} / \mathrm{Si}$ wafer. CVD graphene is transferred on top of the SWNTs layer using the poly(methyl methacrylate) supported procedures. Electrodes are patterned by standard photolithography. Different metal composition ( $\mathrm{Ti} / \mathrm{Au}$ and $\mathrm{Pd} / \mathrm{Au}$ ) are subsequently deposited. The
}

graphene channel is patterned by another photolithography and oxygen plasma etching.

Photoresponse characterization. For photoresponse characterization, we used 405, 532, 650, 980 and 1,550 $\mathrm{nm}$ laser diodes, respectively. The beam is guided through an optical fiber with a FC/PC ferrule and is subsequently incident onto the channel of the devices without focusing. The beam at the device was measured to be Gaussian-shaped with a diameter of about $300 \mu \mathrm{m}$ at $650 \mathrm{~nm}$ illumination. The area of the channel is $<100 \times 40 \mu \mathrm{m}$. Therefore, when calculating the photoresponsivity, we used only the portion of the incident light intensity that overlaps with the channel area. The electrical measurements were carried out in a closed cycle cryogenic probe station under vacuum $\left(10^{-6}\right.$ torr $)$ at room temperature and the data were collected by a Keithley- 4200 semiconductor parameter analyzer. The temporal photoresponse was measured at $173 \mathrm{~K}$ for suppressing the outside interference.

AFM measurements. AFM measurements were performed using an Asylum Research Cypher AFM operating at room temperature and ambient conditions.

Raman and optical absorption spectra. Raman measurements were performed on a Horiba Jobin Yvon LabRAM HR 800 system using a $514 \mathrm{~nm}$ excitation laser operating at $1 \mathrm{~mW}, \times 100$ objective lens with about $1-\mu \mathrm{m}$ diameter spot size, and 1,800 lines per mm grating with about $0.45 \mathrm{~cm}^{-1}$ spectral resolution. Optical absorption spectrum was measured using a Shimadzu UV-vis-NIR UV-3600 spectrophotometer.

\section{References}

1. Geim, A. \& Grigorieva, I. Van der Waals heterostructures. Nature 499, 419-425 (2013).

2. Lv, R., Cruz-Silva, E. \& Terrones, M. Building complex hybrid carbon architectures by covalent interconnections: graphene-nanotube hybrids and more. ACS Nano 8, 4061-4069 (2014).

3. Dimitrakakis, G. K., Tylianakis, E. \& Froudakis, G. E. Pillared graphene: a new 3-D network nanostructure for enhanced hydrogen storage. Nano Lett. 8, 3166-3170 (2008).

4. Paul, R. K. et al. Synthesis of a Pillared Graphene Nanostructure: A Counterpart of Three-Dimensional Carbon Architectures. Small. 6, 2309-2313 (2010).

5. Zhu, Y. et al. A seamless three-dimensional carbon nanotube graphene hybrid material. Nat. Commun. 3, 1225 (2012).

6. Lin, X. et al. Development of an ultra-thin film comprised of a graphene membrane and carbon nanotube vein support. Nat. Commun. 4, 2920 (2013).

7. Yan, Z. et al. Rebar Graphene. ACS Nano 8, 5061-5068 (2014).

8. Koppens, F. H. L., Avouris, M., Ph., Ferrari, A. C., Vitiello, M. S. \& TPolini, M. Photodetectors based on graphene, other two-dimensional materials and hybrid systems. Nat. Nanotechnol. 9, 780-793 (2014).

9. Avouris, P., Freitag, M. \& Perebeinos, V. Carbon-nanotube photonics and optoelectronics. Nat. Photon. 2, 341-350 (2008).

10. Mueller, T., Xia, F. \& Avouris, P. Graphene photodetectors for high-speed optical communications. Nat. Photon. 4, 297-301 (2010).

11. Xia, F., Mueller, T., Lin, Y., Valdes-Garcia, A. \& Avouris, P. Ultrafast graphene photodetector. Nat. Nanotechnol. 4, 839-843 (2009).

12. Echtermeyer, T. J. et al. Strong plasmonic enhancement of photovoltage in graphene. Nat. Commun. 2, 458 (2011).

13. Zhang, Y. et al. Broadband high photoresponse from pure monolayer graphene photodetector. Nat. Commun. 4, 1811 (2013).

14. Engel, M. et al. Light-matter interaction in a microcavity-controlled graphene transistor. Nat. Commun. 3, 906 (2012).

15. Wang, X., Cheng, Z., Xu, K., Tsang, H. K. \& Xu, J. B. High-responsivity graphene/silicon-heterostructure waveguide photodetectors. Nat. Photon. 7, 888-891 (2013).

16. Cao, Y. et al. Ultra-broadband photodetector for the visible to terahertz range by self-assembling reduced graphene oxide-silicon nanowire array heterojunctions. Small. 10, 2345-2351 (2014).

17. Sun, Z. et al. Infrared photodetectors based on CVD-grown graphene and $\mathrm{PbS}$ quantum dots with ultrahigh responsivity. Adv. Mater. 24, 5878-5883 (2012).

18. Konstantatos, G. et al. Hybrid graphene-quantum dot phototransistors with ultrahigh gain. Nat. Nanotechnol. 7, 363-368 (2012).

19. Iijima, S. \& Ichihashi, T. Single-shell carbon nanotubes of $1 \mathrm{~nm}$ diameter. Nature 363, 603-605 (1993).

20. Saito, R., Fujita, M., Dresselhaus, G. \& Dresselhaus, M. S. Electronic structure of chiral graphene tubules. Appl. Phys. Lett. 60, 2204-2206 (1992).

21. Kataura, H. et al. Optical properties of single-wall carbon nanotubes. Synth. Met. 103, 2555-2558 (1999).

22. Dürkop, T., Getty, S., Cobas, E. \& Fuhrer, M. Extraordinary mobility in semiconducting carbon nanotubes. Nano Lett. 4, 35-39 (2004). 
23. Wang, F., Dukovic, G., Brus, L. E. \& Heinz, T. F. The optical resonances in carbon nanotubes arise from excitons. Science 308, 838-841 (2005).

24. Paulus, G. L. C. et al. Charge transfer at junctions of a single layer of graphene and a metallic single walled carbon nanotube. Small. 9, 1954-1963 (2013).

25. Gabor, N. M., Zhong, Z., Bosnick, K., Park, J. \& McEuen, P. L. Extremely efficient multiple electron-hole pair generation in carbon nanotube photodiodes. Science 325, 1367-1371 (2009).

26. Itkis, M. E., Borondics, F., Yu, A. \& Haddon, R. C. Bolometric infrared photoresponse of suspended single-walled carbon nanotube films. Science 312, 413-416 (2006).

27. Gao, M. et al. Carbon nanotube-graphene junctions studied by impedance spectra. Appl. Phys. Lett. 106, 051601 (2015).

28. Strano, M. S. et al. Electronic structure control of single-walled carbon nanotube functionalization. Science 301, 1519-1522 (2003).

29. Gupta, A., Chen, G., Joshi, P., Tadigadapa, S. \& Eklund, P. Raman scattering from high-frequency phonons in supported n-graphene layer films. Nano Lett. 6, 2667-2673 (2006)

30. Grimme, S., Mück-Lichtenfeld, C. \& Antony, J. Noncovalent interactions between graphene sheets and in multishell (hyper) fullerenes. J. Phys. Chem. C 111, 11199-11207 (2007).

31. Pei, T. et al. Electronic transport in single-walled carbon nanotube/graphene junction. Appl. Phys. Lett. 99, 113102 (2011).

32. Tans, S. J., Verschueren, A. R. \& Dekker, C. Room-temperature transistor based on a single carbon nanotube. Nature 393, 49-52 (1998).

33. Bezryadin, A., Verschueren, A., Tans, S. \& Dekker, C. Multiprobe transport experiments on individual single-wall carbon nanotubes. Phys. Rev. Lett. 80, 4036 (1998).

34. Buldum, A. \& Lu, J. P. Contact resistance between carbon nanotubes. Phys. Rev. B 63, 161403 (2001)

35. Stahl, H., Appenzeller, J., Martel, R., Avouris, P. \& Lengeler, B. Intertube coupling in ropes of single-wall carbon nanotubes. Phys. Rev. Lett. 85, 5186 (2000).

36. Lin, Y.-C. et al. Graphene annealing: how clean can it be? Nano Lett. 12, 414-419 (2011)

37. Kim, S. H. et al. Carbon nanotube and graphene hybrid thin film for transparent electrodes and field effect transistors. Adv. Mater. 26, 4247-4252 (2014).

38. Echtermeyer, T. J. et al. Photo-thermoelectric and photoelectric contributions to light detection in metal-graphene-metal photodetectors. Nano Lett. 14, 3733-3742 (2014)

39. Kim, S. et al. Realization of a high mobility dual-gated graphene field-effect transistor with $\mathrm{Al}_{2} \mathrm{O}_{3}$ dielectric. Appl. Phys. Lett. 94, 062107-062107 (2009).
40. Wang, F. et al. Wideband-tuneable, nanotube mode-locked, fibre laser. Nat. Nanotechnol. 3, 738-742 (2008).

41. Yang, F. et al. Chirality-specific growth of single-walled carbon nanotubes on solid alloy catalysts. Nature 510, 522-524 (2014).

\section{Acknowledgements}

This work was supported in part by National Key Basic Research Program of China 2014CB921101, 2011CB301900, 2013CBA01604; National Natural Science Foundation of China 61378025, 61450110087, 61427812, 61274102, 61325020, 61261160499, 61504056; Natural Science Foundation of Jiangsu Province BK20140054. Jiangsu Province Shuangchuang Team Program. Y.D.L. acknowledges funding of the China Postdoctoral Science Foundation 2014M551558 and Jiangsu Planned Projects for Postdoctoral Research Funds 1402028B.

\section{Author contributions}

F.W., X.M.W. and R.Z. conceived and supervised the project. Y.D.L., Y.L. and X.Z.W. performed the photocurrent measurements. Y.D.L. and E.F. carried out sample preparation and device fabrication. X.L. and X.R.W. performed the AFM measurement F.W., X.M.W., Y.X, Y.S. and R.Z. co-wrote the paper with all authors contributing to the discussion and preparation of the manuscript.

\section{Additional information}

Supplementary Information accompanies this paper at http://www.nature.com/ naturecommunications

Competing financial interests: F.W., Y.D.L., Y.L, Y.X. and R.Z have filed a Chinese patent based on this work (application no. 201410265599.1).

Reprints and permission information is available online at http://npg.nature.com/ reprintsandpermissions/

How to cite this article: Liu, Y. et al. Planar carbon nanotube-graphene hybrid films for high-performance broadband photodetectors. Nat. Commun. 6:8589 doi: 10.1038/ ncomms9589 (2015)

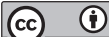

This work is licensed under a Creative Commons Attribution 4.0 International License. The images or other third party material in this article are included in the article's Creative Commons license, unless indicated otherwise in the credit line; if the material is not included under the Creative Commons license, users will need to obtain permission from the license holder to reproduce the material To view a copy of this license, visit http://creativecommons.org/licenses/by/4.0/ 